$Q_{1}$ est l'ensemble qu'on obtient en adjoignant à $P_{1}$ le point 2.

$Q_{1}$ est formé des nombres $0,2, \frac{1}{n}, 2+\frac{1}{n}$ et $\left.4+{ }_{n}^{1}(n=1,2, \ldots)^{1}\right)$.

La démonstration que les cinq ensembles ainsi définis satisfont aux conditions imposées n'offre pas de difficultés.

1) M. Si erpiński désigne $Q_{1}$ par $H_{1}, P_{1}$ par $H_{2}$ et $P_{3}$ par $H_{3}$ ot ácrit (1. c., p. 123) que les types $d H_{1}$ et $d H_{2}$ sont des précédents pour lo type $d H_{3}$ : an lieu de $d H_{1}$ doit $\mathrm{y}$ être éridemment $d Q_{2}$.

\section{Über additive Massfunktionen in abstrakten Mengen.}

Von

\section{S tef a n B a a ch (Lwów).}

In einer vor kurzem gemeinsam mit Herrn Kuratowski veröffentlichten Note $\left.{ }^{1}\right)$ haben wir, unter Voraussetzung der Richtigkeit der Kontinuumhypothese $2 s_{0}=\boldsymbol{N}_{1}$, die folgende Verallgemeinerung eines bekannten Resultats von Herrn Vitali bewiesen:

Es gibt keine total additive nicht identisch verschwindende Mengenfunktion $m(X)$, welche jeder Teilmenge $X$ der Strecke $(0,1)$ eine reelle Zahl zuordnet und für alle aus nur einem Punkte bestehenden Mengen den Wert Null annimmt.

In diesem Satze kann die Strecke $(0,1)$ durch eine beliebige Menge von der Mächtigkeit des Kontinuums ersetzt werden. Die Funktion $m(X)$, welche wir wegen ihrer geometrischen Bedeutung eine Massfunktion, oder auch ein Mass von $X$ nennen, darf auch negative Werte annehmen.

In der vorliegenden Note beweisen wir einen allgemeineren, fur Mengen von beliebiger Măchtigkeit gültigen Satz, wobei wir die Richtigkeit der sog. Hypothese der Alephs, d. h. das Bestehen der Gleichung

$$
2 \mathrm{~s}_{\xi}=\mathrm{s}_{\xi+1}
$$

für beliebige Ordnungszahlen $\xi$ voraussetzen. Um diesen Satz aussprechen zu können, müssen wir zunăchst den Begriff der Massfunktion allgemein definieren.

Wir sagen, dass in einer Menge $E$ eine additive Massfunktion

1) S. Banach et C. Kuratowaki Sur une généralisation du probleme de la mesure Fund. Math. XIV (1929), pp. 127-131. 
definiert ist, falls jeder Teilmenge $G$ von $E$ eine $Z a b l|G|$ zugeordnet ist, welche folgende Bedingungen erfullt:

1. $|G|$ ist nicht identisch null fur alle $G$.

2. Es ist $\left|G_{1}+G_{2}\right|=\left|G_{1}\right|+\left|G_{2}\right|$, falls $G_{1} . G_{2}=0$.

3. Besteht $G$ aus einem einzigen Element von $E$, so ist $|G|=0$.

Eine Massfunktion ist nadditiv mit der Machtigkeit $s_{\xi}{ }{ }$, wenn für disjunkte Mengen $G_{\eta}$ stets

$$
\left|\sum G_{\eta}\right|=\sum\left|G_{\eta}\right| . \quad\left(0<\eta<\omega_{\xi}\right)
$$

Eine Massfunktion ist vom Typus $\kappa_{\xi}$, falls sie mit jeder Machtigkeit kleiner als $\aleph_{\xi}$, nicht aber mit der Măchtigkeit $\aleph_{\xi}$ additiv ist.

Bemerlkung 1. Wenn eine Massfunktion mit einer Muchtigkeit $\aleph_{\xi} \geqslant \aleph_{0}$ additiv ist, so ist jede Klasse disjunkter Teilmengen von $E$ deren jede eine Teilmenge mit von Null verschiedenem Masse enthält, höchstens abzăhlbar.

Bemerkung 2. Wenn die Menge $E$ von der Muchtigkeit $\aleph_{\xi}$ ist, so ist jede in $E$ erklarte Massfunktion höchstens vom Typus $\aleph_{\xi}$. Anderenfalls wäre namlich das Mass einer beliebigen Teilmenge von $E$ gleich der Summe der Masse ihrer Elemente, d. h. gleich Null.

Wir formulieren nunmehr unseren Satz:

Wenn in einer Menge E eine Massfunletion vom Typus $s_{\xi}$ sich definieren lässt, so ist $\boldsymbol{N}_{\xi}$ eine unerreichbare Kardinalzahl ${ }^{1}$ ).

Zum Beweise setzen wir zunuchst voraus, dass die Menge $E$ die Măchtigkeit $\kappa_{\xi}$ besitzt.

Nach der Bemerkung 2. kommt jeder Teilmenge von $k$ deren Mächtigkeit kleiner als $\boldsymbol{N}_{\xi}$ ist, das Mass Null zu. Daher enthalt $E^{r}$ eine Teilmenge von der Muchtigkeit $s_{\xi}$ deren Mass von Null verschieden ist. Da es hier nur auf die Machtigkeit ankommt, durfen wir annehmen, dass die Menge $E$ selbst ein von Null verschiedenes Mass besitzt. Wir führen den Beweis indirekt, nehmen also an, dass $\aleph_{\xi}$ erreichbar ist. Dann sind zwei Fălle zu unterscheiden, je nachdem $\aleph_{\xi}$ eine Grenzzahl ist oder nicht.

1) Eine Kardinalzahl $\aleph_{\alpha}$ nennen wir unerreichbar, falls sie eine Grenzzahl ist und sich nicht, ale Summe von Mengen, welche von Mulchtigkeit $\left\langle\aleph_{\alpha}\right.$ ind und deren Anzahl $<\aleph_{\alpha}$ ist, darstellen Illsst; d. h. die Anfangazahl $\varphi_{\alpha}$ ist regular und $\alpha$ ist eine Limeszahl.

Die kleinste unerreichbare Kardinalzahl ist $\aleph_{0}$. Es ist nicht bekannt, ob es noch andere gibt.
Es sei $\kappa_{\xi}$ eine (erreichbare) Grenzzahl. Die Menge $E$ lassst sich als Summe disjunkter Mengen $\left\{G_{\eta}\right\}$ in der Anzahl $<\aleph_{\xi}$, welche alle von kleinerer Măchtigkeit als $\aleph_{\xi}$ sind, darstellen. Da jede dieser Mengen als Summe ihrer Elemente betrachtet werden kann und unsere Massfunktion dem Typus $\kappa_{\xi}$ angehört, haben alle diese Mengen das Mass Null, also auch die Menge $E$, gegen die Voraussetzung.

Es sei jetzt $\left.s_{\xi}(\xi>1)^{1}\right)$ keine Grenzzahl. Wir bezeichnen mit $A$ die Klasse aller Folgen

$$
\left\{\alpha_{\eta}\right\} \quad\left(0<\eta<\omega_{\xi-1}\right)
$$

wo die $\alpha_{\eta}$ Ordinalzahlen kleiner als $\omega_{1}$ sind. Sind $\left\{\alpha_{\eta}\right\}$ und $\left\{\beta_{\eta}\right\}$ zwei Folgen aus $A$, so schreiben wir

$$
\left\{\alpha_{\eta}\right\}<\left\{\beta_{\eta}\right\}
$$

wenn fur alle $\eta, \alpha_{\eta}<\beta_{\eta}$ ist. Die Machtigkeit von $A$ ist offenbar

$$
\kappa_{1}^{N_{\xi-1}}=\mathrm{s}_{\xi} \text {. }
$$

Wir nehmen an, dass die Menge $A$ nach dem Typus $\omega_{\xi}$ wohlgeordnet ist. Wir entfernen nun jedes Element $\left\{\alpha_{\eta}\right\}$, tür welches ein in $A$ fruher vorkommendes Element $\left\{\beta_{\eta}\right\}$ existiert, derart, dass $\left\{\alpha_{\eta}\right\}<\left\{\beta_{\eta}\right\}$ und bezeichnen mit $\bar{E}$ die Menge der ubrigen Elemente von $A$. Diese Menge $\bar{E}$ besitzt folgende zwei Eigenschaften:

1. Sie ist von der Marchtigkeit $\aleph_{\xi}$.

2. Ist $\left\{a_{\eta}\right\}$ ein Element aus $A$, so ist die Menge derjenigen Elemente von $\bar{E}$, welche kleiner als $\left\{\alpha_{\eta}\right\}$ sind, von einer kleineren Mächtigkeit als $\mathrm{s}_{\xi}$.

- Unter der Annahme, dass die Măchtigkeit von $\bar{E}$ kleiner als $\aleph_{\xi}$ ist, kann $\bar{E}$ als eine wohlgeordnete Menge von Folgen $\left\{\beta_{\eta}^{\vartheta}\right\}, 0<\vartheta<\omega_{\xi-1}$ dargestellt werden. Sei $\alpha_{\eta}=\beta_{\eta}^{\eta}+1$; alsdann existiert keine Folge $\left\{\beta_{\eta}\right\}$ aus $\bar{E}$ für welche $\left\{\alpha_{\eta}\right\} \leqslant\left\{\beta_{\eta}\right\}$ ist, - im Widerspruch mit der Definition der Menge $\bar{E}$.

Ist ferner $\left\{\beta_{\eta}\right\}<\left\{\alpha_{\eta}\right\}$ und $\left\{\beta_{\eta}\right\} \subset \bar{E}$, so kommt $\left\{\beta_{\eta}\right\}$ früher in $\boldsymbol{A}$ vor als $\left\{\alpha_{\eta}\right\}$. Die Menge derjenigen Elemente, welche in $A$ fruher als $\left\{\alpha_{\eta}\right\}$ vorkommen, ist aber von einer kleineren Mrchtigkeit als $\aleph_{\xi}$.

1) Für den Beweis im Falle $\xi=1$ verweisen wir auf die zitierte Note. 
Es sei $\alpha<\omega_{1}$. Wir bezeichnen mit $\bar{E}_{\alpha}^{\eta}$ die Menge derjenigen Folgen $\left\{\beta_{\eta}\right\}$ von $\bar{E}$, fur welche $\beta_{\eta}=\alpha$.

Es ist klar, dass

$$
\bar{E}=\sum_{0<\alpha<\omega} \bar{E}_{\alpha}^{\eta}
$$

Da nach 1. die Mengen $E$ und $\bar{E}$ von gleicher Machtigkeit sind, so lässt sich in $\bar{E}$ eine Massfunktion vom Typus $s_{\xi}$ erklaren derart, dass $|\bar{E}| \neq 0$ ist. Die Menge aller $\alpha$ besitzt die Muchtigkeit $\aleph_{1}$. Daher gibt es nach der Bemerkung 1. eine Ordnungazahl $\alpha_{\eta}<\omega_{1}$ derart, dass fur jedes $\eta$

1. $\left|\bar{E}_{\alpha}^{\eta}\right|=0$ fur $\alpha \geqslant \alpha_{\eta}$,

2. $|G|=0$ fur jede Teilmenge $G$ eines $\overline{E_{\alpha}}\left(\alpha \geqslant \alpha_{\eta}\right)$.

Wir setzen

$$
\bar{E}^{\prime}=\sum_{0<\eta<\omega_{\xi}-1} \sum_{\alpha \geq \alpha} \bar{E}_{\alpha^{\eta}}
$$

Da die Teilmengen von $\bar{E}_{a}^{\eta}$ vom Mass Null sind und die obige Summe aus Mengen von der Mrchtigkeit $\aleph_{\xi-1}$ besteht, so ist $\left|\bar{E}^{\prime}\right|=0$.

Die Menge $\bar{E}-\overline{E^{\prime}}$ enthalt nur diejenigen Folgen von $\bar{E}$, welche kleiner als $\left\{\alpha_{\eta}\right\}$ sind. Die Anzahl dieser Folgen ist, nach der zweiten "Eigenschatt der Menge $\bar{E}$, höchstẹns $\aleph_{\xi-1}$. Daher ist $\left|\bar{E}-\overline{E^{\prime}}\right|=0$, also

$$
|\bar{E}|=\left|\overline{E^{\prime}}\right|+\left|\bar{E}-\overline{E^{\prime}}\right|=0
$$

- gegen die Voraussetzung.

Damit ist der Beweis unseres Satzes für den Fall, dass die Menge $E$ die Machtigkeit $\aleph_{\xi}$ besitzt, erledigt. Um daraus den Beweis für den allgemeinen Fall abzuleiten, bemerken wir, dass aus der Voraussetzung unseres Satzes leicht die Existenz einer Klasse disjunkter Teilmengen $E_{\eta}\left(0<\eta<\omega_{\xi}\right)$ von $E$ folgt, fur welche:

$$
\left|\sum_{0<\eta<\omega_{\xi}} E_{\eta}\right| \neq \sum_{0<\eta<\omega_{\xi}}\left|E_{\eta}\right|
$$

Nach der Bemerkung 1. haben nur abzüblbar viele Mengen $E_{\eta}$ ein von Null verschiedenes Mass. Wir bezeichnen die ubrigen mit $G_{\eta^{*}}$.
Es ist klar, dass

und

$$
\left|G_{i \mid}\right|=0
$$

ist.

$$
\left|\sum G_{\eta}\right| \neq \sum\left|G_{\eta}\right|=0
$$

Wir bezeichnen mit $W$ die Menge aller Ordnungszahlen, welche kleiner als $\omega_{\xi}$ sind. In dieser Menge, deren Măchtigkeit $\kappa_{\xi}$ betragt, definieren wir eine Massfunktion vom Typus $\kappa_{\xi}$ folgendermassen: Ist $\boldsymbol{M}$ eine Teilmenge von $W$, so setzen wir

$$
|M|=\left|\sum G_{\eta}\right|
$$

wobei die Summe uber alle Ordnungszahlen von $M$ erstreckt ist. Da nach der vorletzten Relation $|W| \neq 0$ ist, so folgt aus unserem frtheren Ergebnis, dass $\kappa_{\xi}$ eine unerreichbare Kardinalzahl ist, w. z. b. w.

Auf Grund dieses Satzes beweist man leicht mit Hilfe der Bemerkung 2. folgendes Korollar:

Es sei $\bar{\aleph}$ die kleinste unerreichbare Kardinalzahl, welche grösser als $\aleph_{0}$ ist. Wenn $E$ eine Menge von einer kleineren Mächtigkeit als


funktion vom Typus $\aleph_{0}, d$. $h$. es ist nicht immer

$$
\left|\sum_{n=1}^{\infty} E_{n}\right|=\sum_{n=1}^{\infty}\left|E_{n}\right|
$$

für disjunkte Mengen $E_{n}$. 